\title{
Current techniques in postmortem imaging with specific attention to paediatric applications
}

\author{
Tessa Sieswerda-Hoogendoorn • Rick R. van Rijn
}

Received: 12 October 2009/Revised: 9 November 2009/Accepted: 16 November 2009/Published online: 16 December 2009

(C) The Author(s) 2009. This article is published with open access at Springerlink.com

\begin{abstract}
In this review we discuss the decline of and current controversies regarding conventional autopsies and the use of postmortem radiology as an adjunct to and a possible alternative for the conventional autopsy. We will address the radiological techniques and applications for postmortem imaging in children.
\end{abstract}

Keywords Autopsy Radiology $\cdot$ Child · Forensics

\section{Introduction}

The number of autopsies has been declining worldwide. In a study performed at the Agency for Healthcare Research and Quality, Shojania et al. [1] found an autopsy rate in the United States below 6\%, compared with $40-50 \%$ a few decades ago. This trend is supported by other sources verified by the authors [2]. In a review by Burton and Underwood [3], similar rates were found in other Western countries as well (Table 1).

The paediatric and neonatal autopsy rates have always been higher than those in adults [4]. A general trend towards declining paediatric autopsy rates has been noticed as well $[5,6]$. The reason for this decline is twofold-a decrease in willingness of the attending physician to ask

\footnotetext{
T. Sieswerda-Hoogendoorn • R. R. van Rijn $(\bowtie)$

Department of Radiology,

Academic Medical Centre Amsterdam,

Meibergdreef 9, 1105 AZ,

Amsterdam Zuid-Oost, Netherlands

e-mail: r.r.vanrijn@amc.uva.nl

T. Sieswerda-Hoogendoorn • R. R. van Rijn

Department of Pathology and Toxicology,

Netherlands Forensic Institute,

The Hague, Netherlands
}

permission for an autopsy in combination with a decrease in parental willingness to give consent.

Little research has been done on the diminished willingness of doctors to ask permission to perform an autopsy. In a study by Snowdon et al. [7], neonatologists said that although autopsies are important, they believed it was secondary to parental needs. They felt especially uncomfortable if the cause of death could be determined without an autopsy. This is supported by several other authors $[8,9]$. Other factors that are mentioned are budget cuts, fear of legal consequences, lack of communication training, and prior negative experiences with poorly performed autopsies or delayed autopsy reports [10].

Refusal of parents/guardians to agree to an autopsy is influenced by several factors, including religion [11, 12], fear of unethical practices (influenced by the Alder Hey scandal in the UK [13-17]), and an increasingly individualistic culture in which personal life and experience precedes gain in scientific knowledge [9]. An important reason for relatives to refuse permission is the feeling that the deceased "has suffered enough" [18].

Many of these reasons have not changed during the last decades and therefore do not explain the decrease in the number of autopsies. Perhaps the most important reason for the decline in autopsies is the fact that, with increasing diagnostic and imaging techniques, both doctors and parents/ guardians are under the assumption that they already know the cause of death. This assumption is known not to be true for adults in about one-third of the cases [19-21]. For neonates and children, discrepancies between clinical diagnosis and diagnosis at autopsy are found in about one-quarter of the cases $[6,22]$. The autopsy reveals new information in paediatric deaths in about $44 \%$ of the cases [23].

An alternative for conventional autopsy could be postmortem radiology (also known as virtual autopsy or 
Table 1 The worldwide decline in autopsy rates. Autopsy rate is expressed as a percentage of all deaths. Figures in brackets denote the years in which the data were reported (adapted from Burton and Underwood [3] with permission)
Initial autopsy rate (period)

Subsequent autopsy rate (period)

\begin{tabular}{lcc}
\hline Australia & $21.0 \%(1992-93)$ & $12.0 \%(2002-03)$ \\
France & $15.4 \%(1988)$ & $3.7 \%(1997)$ \\
Hungary & $100 \%(1938-51)$ & $68.9 \%(1990-02)$ \\
Ireland & $30.4 \%(1990)$ & $18.4 \%(1999)$ \\
Jamaica & $65.3 \%(1968)$ & $39.3 \%(1997)$ \\
Sweden & $81.0 \%(1984)$ & $34.0 \%(1993)$ \\
UK & $42.7 \%(1979)$ & $15.3 \%(2001)$ \\
USA & $26.67 \%(1967)$ & $12.4 \%(1993)$ \\
\hline
\end{tabular}

Virtopsy ${ }^{\circledR}$ ) or minimally invasive autopsy (CT and MR imaging followed by ultrasonographic-guided biopsies) (www.virtopsy.com) [13, 24-26]. In this review article we address the radiological techniques, conditions and applications for postmortem imaging in children.

\section{Techniques}

Conventional radiography

Conventional radiology (CR) is the mainstay of postmortem imaging. The radiographs are reported by a paediatric radiologist and emphasis is placed on skeletal development, both with regard to the gestational age as well as the presence of anomalies, such as skeletal dysplasias. In foetuses up to a gestational age of approximately 24 weeks, we make use of the mammography system, as this has a high resolution and exquisitely depicts the foetal skeleton (Fig. 1). In these cases a babygram, which visualizes developmental anomalies of the entire skeletal system in two or more views, is acceptable.

In older foetuses and neonates a direct digital radiography system (Triathlon DR, Oldelft Benelux, the Netherlands) is used. In babies and toddlers postmortem radiography is reserved for cases of sudden infant death syndrome (SIDS) or suspected child abuse. In these cases a full skeletal survey according to either the American College of Radiology or the Royal College of Radiologists should be performed, even if a whole-body CT is obtained [27-29]. In older children ( $>4$ years of age) postmortem CR plays a minor role and is only performed on special indications.

Finally, pathologists may request radiographs of autopsy specimen. These radiographs should preferably be obtained in close cooperation with the attending pathologist and if size permits should be made on a mammography system. These specimen radiographs can yield additional information that initially was not visible on either CR or CT (Fig. 2).

\section{Conventional angiography}

Since conventional autopsy examination of the vascular system is difficult to perform, postmortem angiography could be a useful technique [30]. In most cases postmortem angiography will consist of a single organ study in which the organ can be in situ or removed from the body. Wholebody postmortem conventional angiography has been described in foetuses and neonates [31]. A special technique worth mentioning is the art of cast angiography in which a resin is injected into the vasculature and the tissues are removed by maceration, thus yielding a cast of the vasculature.

\section{Ultrasonography}

The use of US in postmortem imaging, to date, has been limited (Fig. 3) [32, 33]. Implementation is hindered by a relative lack of knowledge about the possibilities of US by (forensic) pathologists. Not only can US be used as an inexpensive imaging method in the absence of CT and/or MRI, but it can also be used to guide biopsy procedures in case of a minimal invasive autopsy (MIA).

\section{Computed tomography}

Postmortem CT is a fast technique that allows imaging of the whole body inside a body bag or coffin. This makes access to the scanner relatively easy and technicians appreciate the fact that they are not confronted with the deceased person. In general, straightforward CT protocols are used. In our hospital we routinely perform these exams and use separate protocols for the brain and the rest of the body (Table 2). The use of 3-D reconstructions can be very illustrative.

One of the clear disadvantages of postmortem CT is the absence of blood flow; this makes CT angiography (CTA) difficult. In the Virtopsy ${ }^{\circledR}$ project, postmortem use of CTA has been explored [34]. Using a pressure-controlled modified heart-lung machine and femoral access, postmortem angiography is a feasible option in specialized centres. 
Fig. 1 A neonate aborted at 14 weeks gestational age. a Antenatal US showed severe dysmorphological changes. Photograph of the foetus shows the fused lower extremity. The insert depicts the size of the foetus in relation to the fingertip of the pathology assistant (arrow). b Radiography, performed on a mammography system, shows a sirenomelia. The skeleton is exquisitely depicted
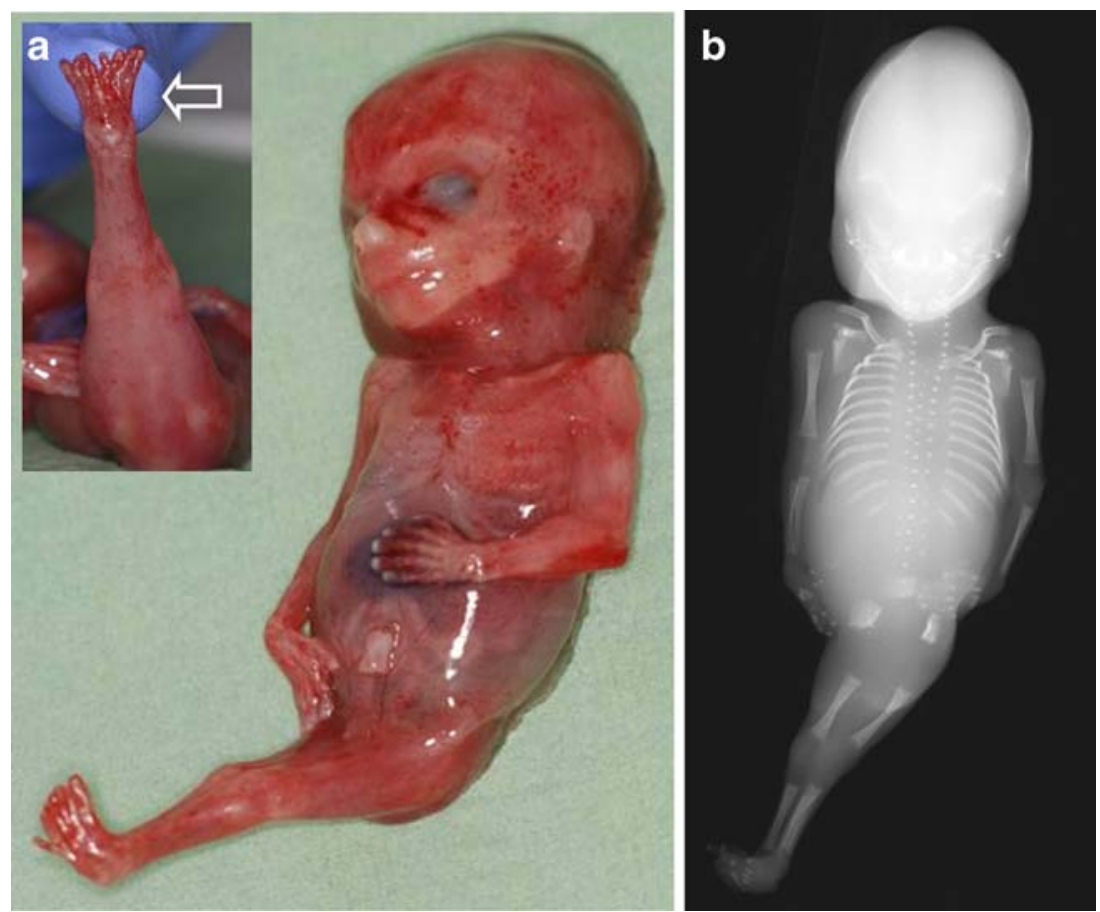

\section{Magnetic resonance imaging}

We feel that CR still has an important role and therefore should be obtained in all cases in which postmortem MRI of foetuses and neonates is performed. In some instances pathology will be difficult, if not impossible, to detect on MRI, whereas CR can be diagnostic (Fig. 4).

The MRI protocol is divided into two separate parts. First of all the neurocranium (in a significant number of cases neuropathology will be present) (Table 3) (Fig. 5). The thorax and abdomen are imaged separately (Table 3) (Fig. 6). The extremities are only imaged upon special

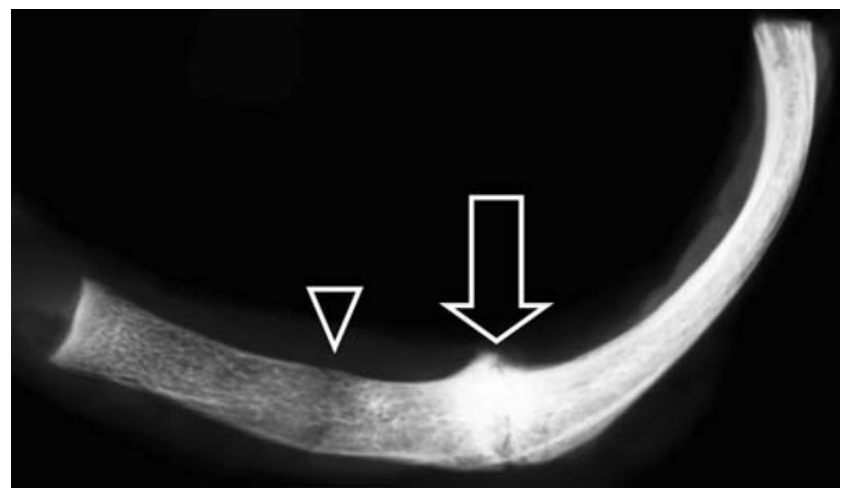

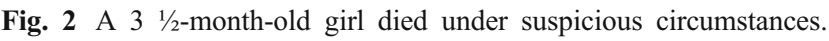
Judicial autopsy was warranted. Contact radiograph (performed on mammography system) shows a fracture of the second rib on the left with consolidation (arrow) but also a fresh fracture (arrowhead). The latter was, even in retrospect, not visible on CR or CT (not shown here) (Reprinted with permission from Bilo RA, Robben SG, van Rijn RR [2009] Forensic aspects of paediatric fractures: differentiating accidental trauma from child abuse. Springer-Verlag, in press) request. In general this protocol can be scanned within a 1-h time frame. Protocols should be fine-tuned if specific questions need to be answered.

To date, in our hospital, we have only performed postmortem MRI in fresh cadavers that can be placed in the bore without problems. However, it is possible to obtain an MRI while the corpse is in the body bag.

Technicians and local guidelines

Before a postmortem radiology service is offered in a department of radiology, the radiological technicians and

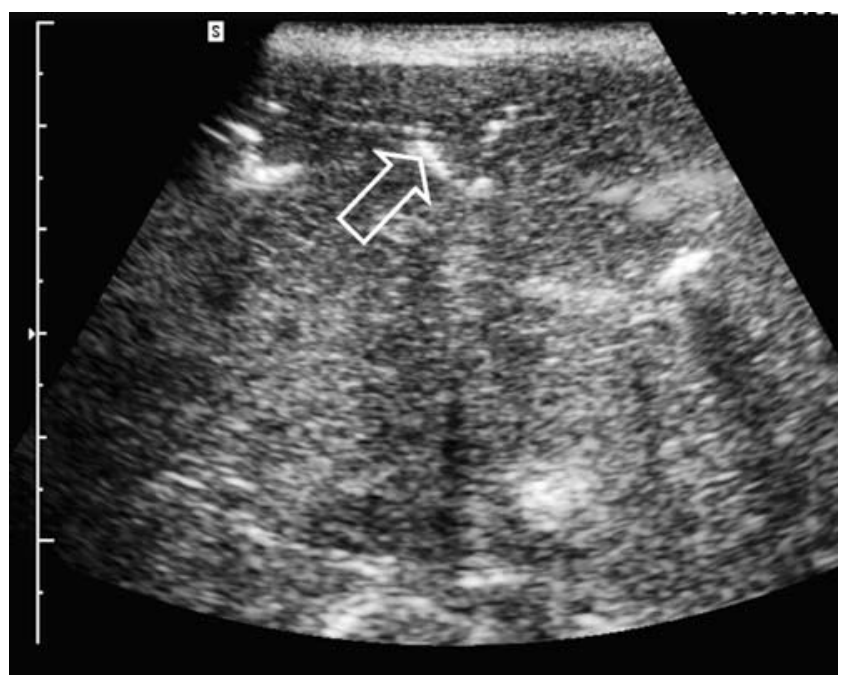

Fig. 3 Postmortem US shows portal air (arrow), a common finding in postmortem imaging 
Table 2 Postmortem CT parameters ${ }^{\mathrm{a}}$

\begin{tabular}{lccccccc}
\hline Anatomical location & $\mathrm{kV}$ & $\mathrm{mAs}$ & Slice thickness $(\mathrm{mm})$ & Increment (mm) & Pitch & Collimation (mm) & Rotation time (sec) \\
\hline Head \& neck & 120 & 285 & 0.9 & 0.45 & 0.392 & $64 * 0,625$ \\
Thorax \& abdomen $^{\mathrm{b}}$ & 120 & 250 & 3.0 & 2.0 & 0.983 & $64 * 0,625$ & 0.75 \\
\hline
\end{tabular}

${ }^{\text {a }}$ Protocol for Philips Brilliance (64 channel CT, Philips Medical Systems, Best, the Netherlands)

${ }^{\mathrm{b}}$ Lower extremities will be scanned upon special request only

In all cases, coronal and sagittal reconstructions, using appropriate kernels, are performed

In selected cases 3-D SSD reconstructions are performed

other involved personnel should be informed. It should be made clear to all involved that postmortem imaging is an important aspect of medical care and that the outcome of the exam can seriously impact the life of parents/guardians.
In our department we have the policy that these exams are performed on a voluntary basis. However, to date none of our technicians has refused to do these exams. Handling of the deceased foetus, neonate or child is done in all instances

Fig. 4 A neonate, born at 41 weeks gestational age, who died shortly after birth. a Antenatal US showed an underdeveloped thorax and short stature (T2-W 3-D, slice thickness: $1 \mathrm{~mm}$, TR: 4000, TE: 80). b Chest radiograph shows a severely constricted thoracic cage, underdeveloped scapulae and flattened vertebrae. c Radiograph of the pelvis shows hypoplastic iliac wings and sciatic notch spurs (arrow). Based on the conventional radiological findings, the diagnosis thanatophoric dysplasia type II is most likely (OMIM \#187601)
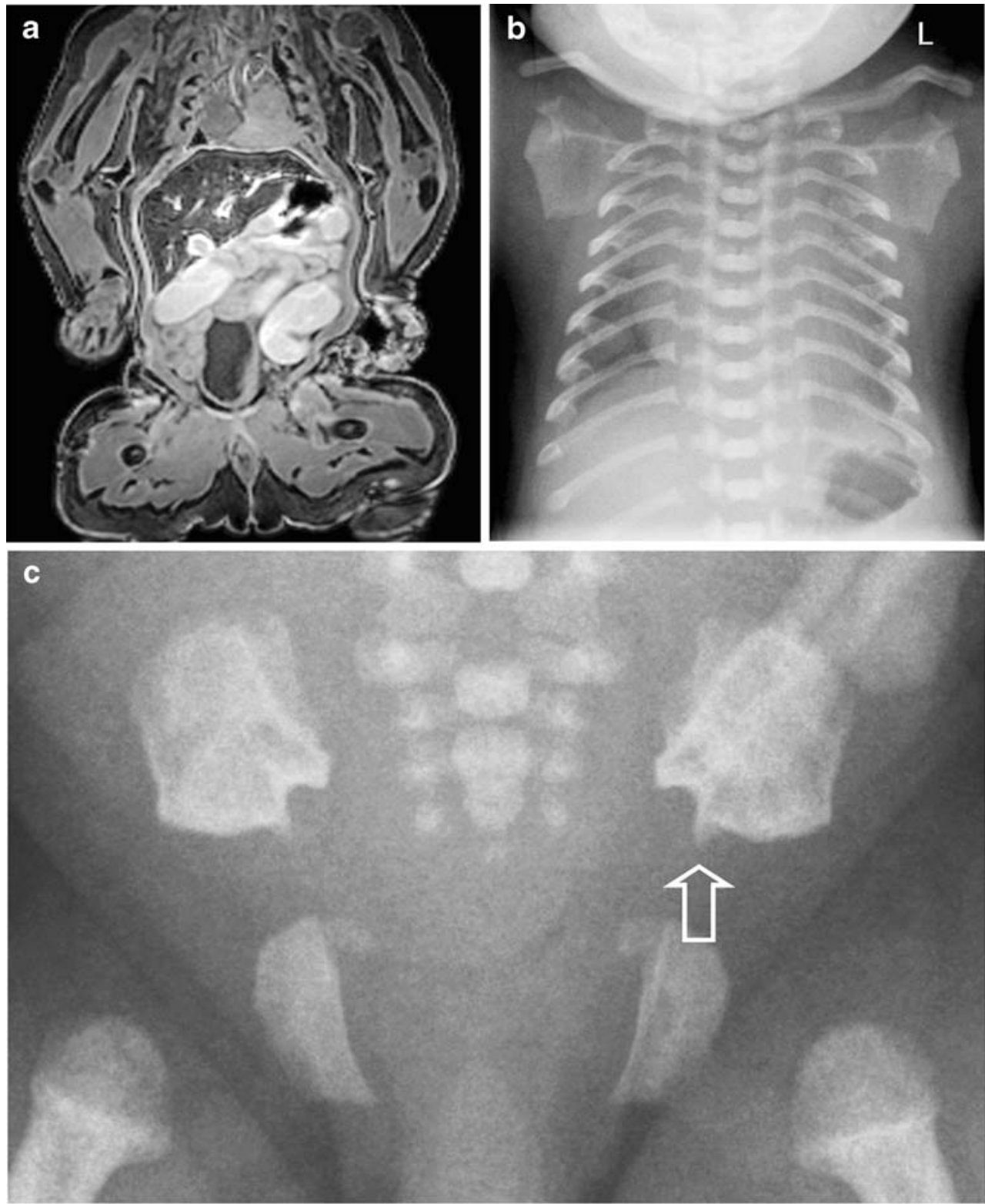
Table 3 Postmortem MRI parameters ${ }^{\mathrm{a}}$

Anatomic location

\begin{tabular}{|c|c|c|c|c|c|c|c|c|}
\hline Head \& neck & Sequence & FoV (mm) & Sl. Thickness (mm) & TR (msec) & TE (msec) & NSA & FA $\left(^{\circ}\right)$ & $\mathbf{A} \mathbf{T}^{\mathbf{b}}(\min )$ \\
\hline Axial & $\mathrm{T} 2$ & 180 & 3 & 4000 & 96 & 4 & 150 & $3: 18$ \\
\hline Sagittal $^{\mathrm{c}}$ & $\mathrm{T} 1-3 \mathrm{D}$ & 256 & 1 & 1990 & 2.92 & 1 & 15 & $4: 06$ \\
\hline Axial $^{\mathrm{d}}$ & Flash 2D & 180 & 3 & 660 & 26 & 2 & 20 & $5: 39$ \\
\hline \multicolumn{9}{|l|}{ Body $^{e}$} \\
\hline Coronal $^{\mathrm{f}}$ & $3 \mathrm{D}-\mathrm{T} 2$ & 300 & 1 & 750 & 108 & 1 & 150 & $8: 26$ \\
\hline Coronal & 3D-T1 & 300 & 3 & 1500 & 13 & 2 & 90 & $6: 27$ \\
\hline
\end{tabular}

${ }^{\text {a }}$ Protocol for Siemens Magnetom Avanto 1.5 T (Siemens, Erlangen, Germany)

b $\mathrm{TA}=$ Acquisition time

${ }^{\mathrm{c}}$ Coronal and axial reconstructions

${ }^{d}$ In case of clinical suspicion of intracranial haemorrhage only

${ }^{\mathrm{e}}$ Lower extremities will be scanned upon special request only

${ }^{\mathrm{f}}$ Axial and sagittal reconstructions

by either the mortuary personnel or the attending paediatric radiologist.

The referring clinician should be aware that postmortem imaging is not a routine procedure and that normal clinical work will have priority over these exams. In general this means that the exams will be done before or after the normal radiology schedule.

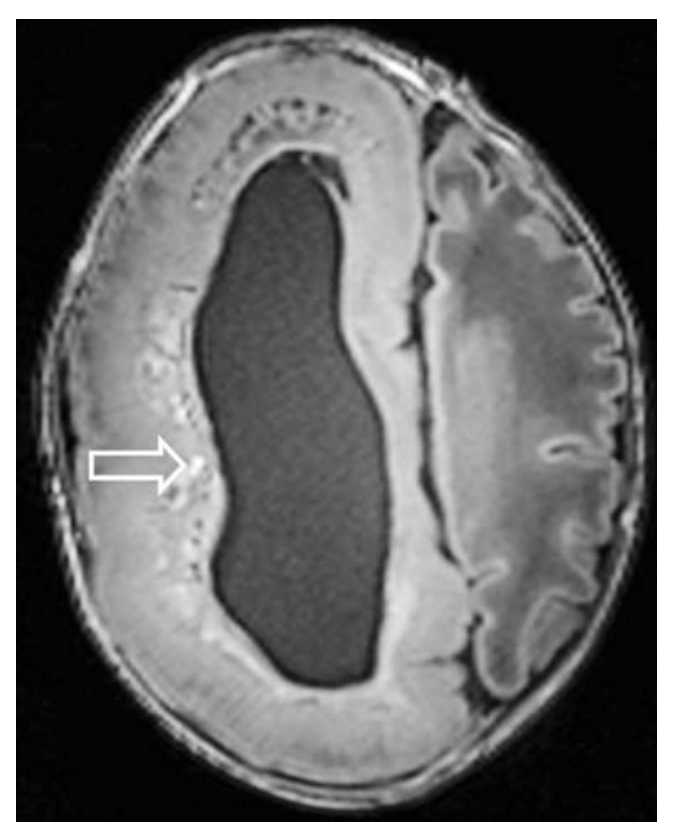

Fig. 5 A neonate aborted at 31 weeks gestational age. Antenatal US showed abnormal brain development. Autopsy was refused by the parents. T1-W MRI shows asymmetrical development of the brain with overgrowth of the right side, in keeping with hemimegalencephaly. On the right side multiple focal hemorrhagic lesions are seen (arrow) (slice thickness: $1 \mathrm{~mm}$, TR: 9, TE: 4,1) [58]

\section{Clinical postmortem radiology}

If postmortem imaging proves to be an adequate alternative for the conventional autopsy, this will probably lead to more investigations of deceased children. This way, major improvements could be made in studying clinically challenging diagnoses, e.g. sudden infant death syndrome. At the present time, postmortem imaging can be an interesting addition to conventional autopsy. Comparison studies show that for certain diagnoses imaging is superior, while in others the cause of death cannot be determined. Most information comes from perinatal (neuro-) imaging and imaging in trauma patients.

\section{Conventional radiography}

It is widely known that conventional radiology plays an important role in the diagnosis of skeletal dysplasias and the detection of fractures in cases of child abuse [35]. Detailed discussion of conventional radiology lies outside the scope of this review as this is a widely accepted and utilized technique [36-38].

\section{Ultrasonography}

To date only a few articles on postmortem US have been published. Uchigasaki et al. [33] state that although CT and MRI can provide much more information than US (especially in decomposed bodies), US is inexpensive and easy to handle, and therefore might provide some information before an autopsy is performed. Farina et al. [32] studied 100 cases in which US and US-guided biopsy were performed [32]. In this study the concordance rate between US and conventional autopsy with regard to the 
Fig. 6 A neonate with a congenital cyanotic heart disease born at 39 weeks. Maximum support and $100 \%$ oxygen did not lead to clinical improvement and the child died. T2-W coronal MRI shows a complete anomalous venous return (arrow) with pulmonary interstitial oedema (insert). A central tendon defect is seen (open arrow) (slice thickness: $2 \mathrm{~mm}$, TR: 5500, TE: 54, FA: $180^{\circ}$ ). b T2-W coronal MRI shows a persistent left superior caval vein (arrow), a dextrocardia and situs intermedius of the liver. Asplenia was also noted
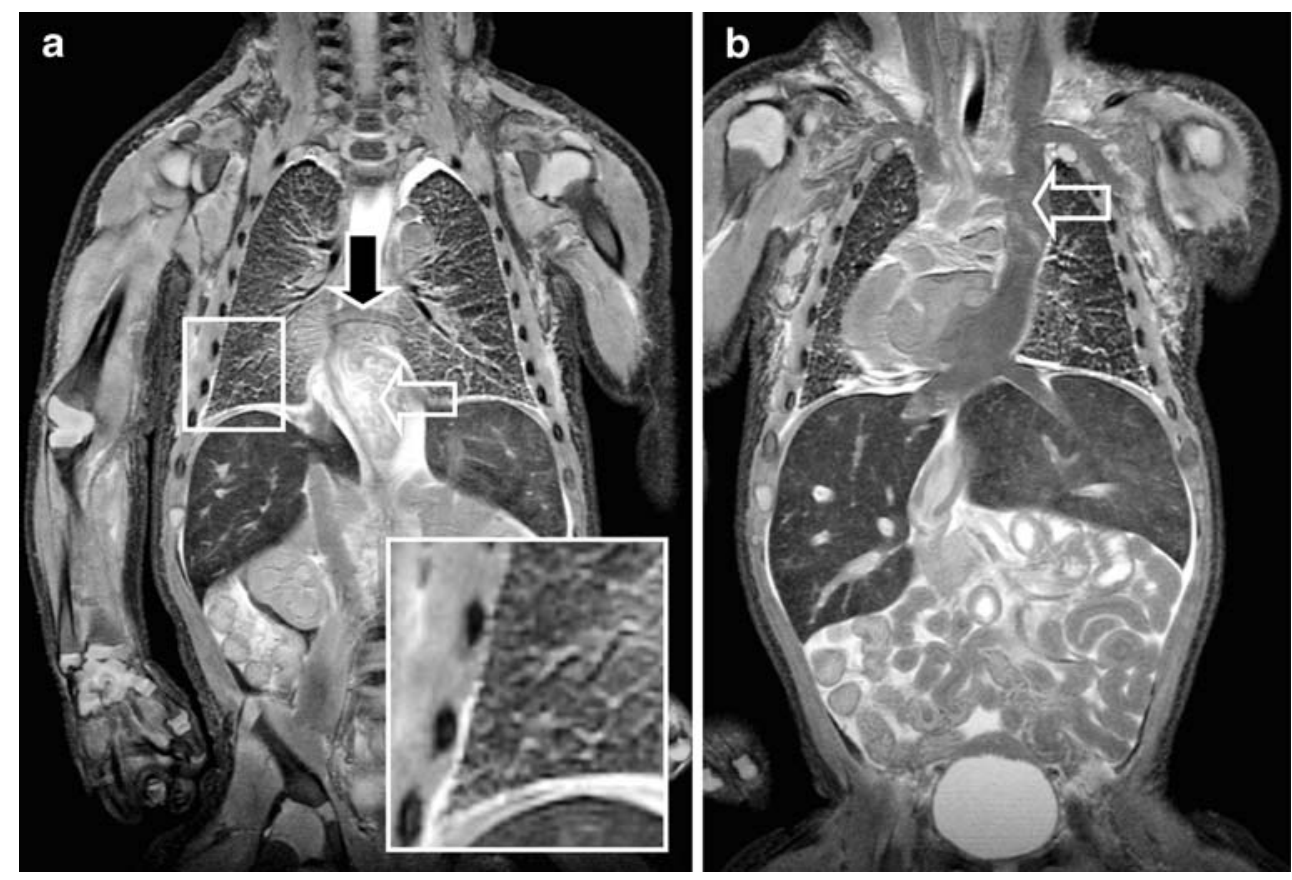

cause of death and the main pathological findings reached $83 \%$.

\section{Computed tomography}

Compared to autopsy $\mathrm{CT}$ is superior in detecting fractures, as it can detect fractures in places that are generally not examined during a conventional autopsy (e.g. the face). Bolliger et al. [39] conclude from their study that CT examination has proven "to be an invaluable tool in three areas of forensic pathology; namely, in the detection and demonstration of fractures, the detection of foreign bodies and the detection of gas" (Fig. 7). Furthermore, 3-D reconstructions can be made, which can be helpful in demonstrating the type of injury during court cases, for example.

\section{Magnetic resonance imaging}

Obtaining permission for an examination of the brain during an autopsy can be especially difficult because it takes several weeks to fixate the brain properly, and many parents/guardians request that all organs be replaced before burial [40]. It is therefore encouraging that several studies have shown that structural anomalies of the brain can be adequately detected with MRI [26, 41]. Griffiths et al. [42] have been examining neuropathology in foetuses and deceased neonates since 2003. In their first series they found complete agreement between MRI and autopsy in 28 of 32 cases. In 2005 they examined more than 200 foetuses and neonates with similar results. They found that MR provides detailed information about all organ systems, except for the heart (Figs. 8 and 9) [13]. Cohen et al. [25] found that although MR is very good in detecting brain and spine anomalies, if it is not combined with the results of autopsy, $71 \%$ of essential information will not be detected. Breeze et al. [43] determined kappa values to assess agreement between MRI and autopsy for different organ systems. They were high for the brain (0.83), moderate for lungs $(0.56)$ and fair for the heart $(0.33)$. The relative inability of postmortem MRI to detect cardiac pathology is described by several other authors [43, 44]. This is a major

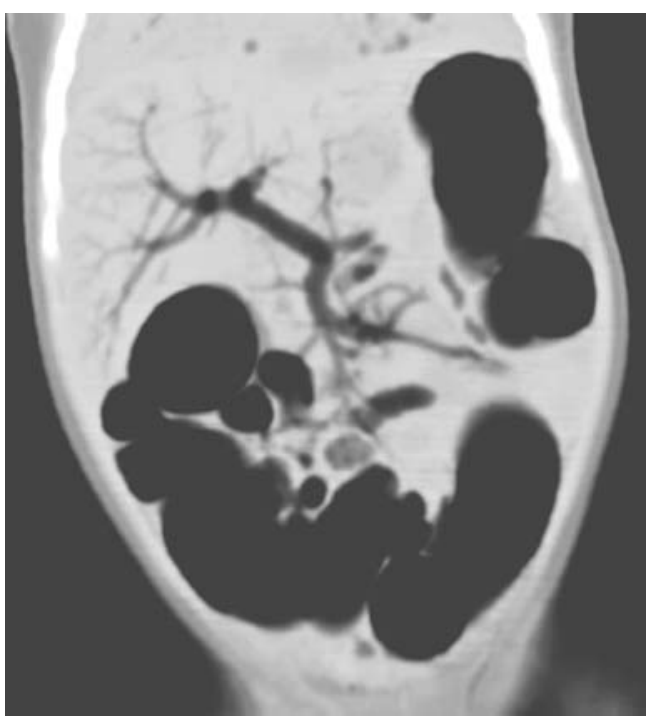

Fig. 7 A 6-month-old boy who died after attempted resuscitation. On postmortem, CT air is seen in all major vessels. On autopsy a positive blood culture for $S$. aureus was found. The cause of death was a fulminant sepsis 


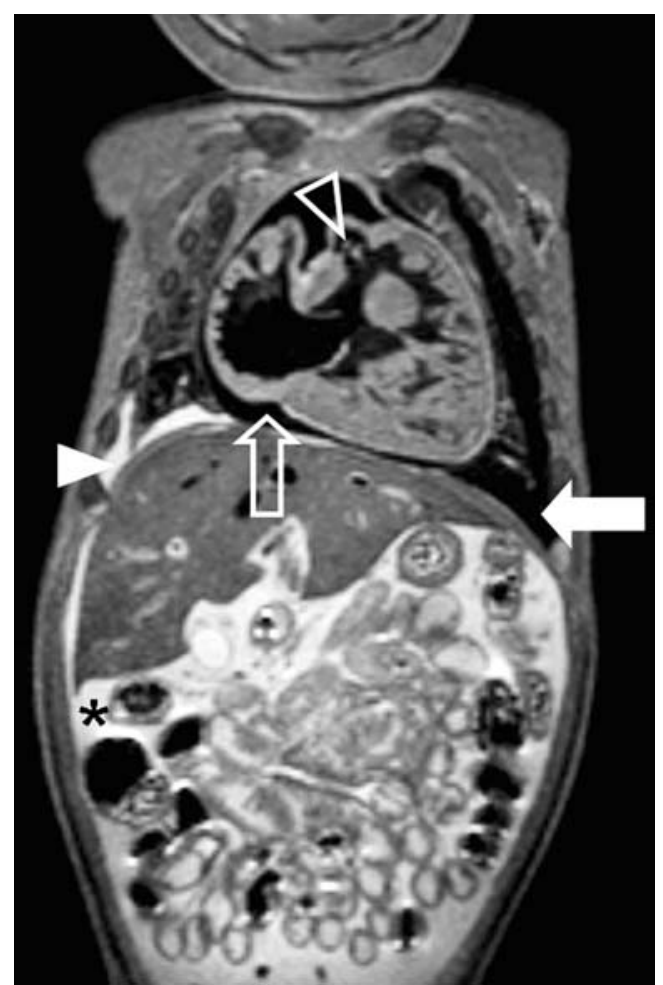

Fig. 8 A 2-month-old neonate presented at the emergency department in severe cardiac and respiratory distress. Resuscitation was unsuccessful. Coronal T2-W MRI shows hematopericard (open arrow), hematothorax (arrow) and a pleural effusion (arrowhead) (slice thickness: $1 \mathrm{~mm}$, TR: 4000, TE: 80 , FA: $90^{\circ}$ ). There is an abberant pulmonary vein draining into the left ventricle (open arrowhead). The abdomen shows ascites (asterisk)

shortcoming because cardiac disease is a major cause of death in the Western world.

The use of ultra-high-field MRI has been evaluated by Thayyil et al. [45], who concluded that "high-field MRI (and ancillary non-invasive post-mortem investigations) provided all the information that could be obtained with invasive autopsy for all internal organs in cases in which the intrauterine retention period was less than 1 week. Moreover, clinically useful information about the brain could be obtained, even in cases in which maceration and autolysis prevented formal neuropathological examination" [45].

\section{Combined CT and MRI}

In a recent study, 30 adult study subjects underwent both minimally invasive autopsy (MIA) and conventional autopsy (CA) [46]. Because of a wide variety of causes of death, it is difficult to draw conclusions in such a small group about the sensitivity and specificity for CA of different organ systems, but the overall agreement on cause of death between MIA and CA was $77 \%$. MIA correctly identified common causes of death, such as pneumonia and sepsis, but failed to demonstrate acute myocardial infarction $(n=4)$. In this study MRI was superior to CT in detecting brain abnormalities and pulmonary embolus. Conversely, CT was superior to MRI for the detection of calcifications and pneumothorax. A head-to-head analysis for CT versus MRI was not presented.

Yen et al. [47] performed a retrospective study on postmortem neuroimaging (mostly in adults) as part of the Virtopsy ${ }^{\circledR}$ project, a Swiss research project that aims to eventually replace the standard autopsy by a virtual one combined with minimally invasive procedures. They found that imaging, compared with autopsy as the gold standard, correctly identified the cause of death in almost $80 \%$ of the cases where the brain was the primary atrium mortis. The overall agreement between CT and MRI was $69 \%$. A closer look at different findings shows big differences between the accuracy of imaging and autopsy for different types of lesions. Autopsy was superior in detecting scalp lesions, intracranial blood layers and contusions less than $3 \mathrm{~mm}$ in thickness, plaque jaunes, dura mater ruptures and brain oedema. Imaging, on the other hand, was better in visualizing gunshot injuries and complex skull fractures and in detecting pneumencephalon, ventricular haemorrhage and facial bone fractures. Furthermore, imaging was better in detecting lesions in a decomposed body. It has to be noted that the radiologists reviewing the CT and MRI data were not particularly trained in the forensic field, but that the outcome of the evaluation depends to a large degree on the previous forensic training of the radiologists [47].

\section{Forensic postmortem radiology}

The word "forensic" comes from the Latin adjective forensis, meaning "of or before the forum." Today "forensic" is interchangeably used with "forensic sciences" and implicitly means "related to the court" or "legal."

The use of radiology in the legal system actually dates to 7 February 1896 when in Montreal, Canada, a radiograph (with an exposure time of $45 \mathrm{~min}$ ) was obtained to locate a bullet lodged in the leg of a gunshot victim. Based on the radiograph the assailant could be convicted [48].

With respect to forensic radiology, one aspect that needs to be addressed is patient confidentiality. Not only are the radiographs part of the evidence, and as such should be kept confidential in order not to compromise the chain of evidence, but there may also be interest from third parties not directly involved in the criminal procedure. It is good practice to store the data anonymously.

In the last few decades radiology has been widely used in forensic sciences; however, in most cases radiologists have not been involved. In contrast to the clinical situation, forensic radiology will in most cases be done in children in whom the cause and manner of death are unclear and 


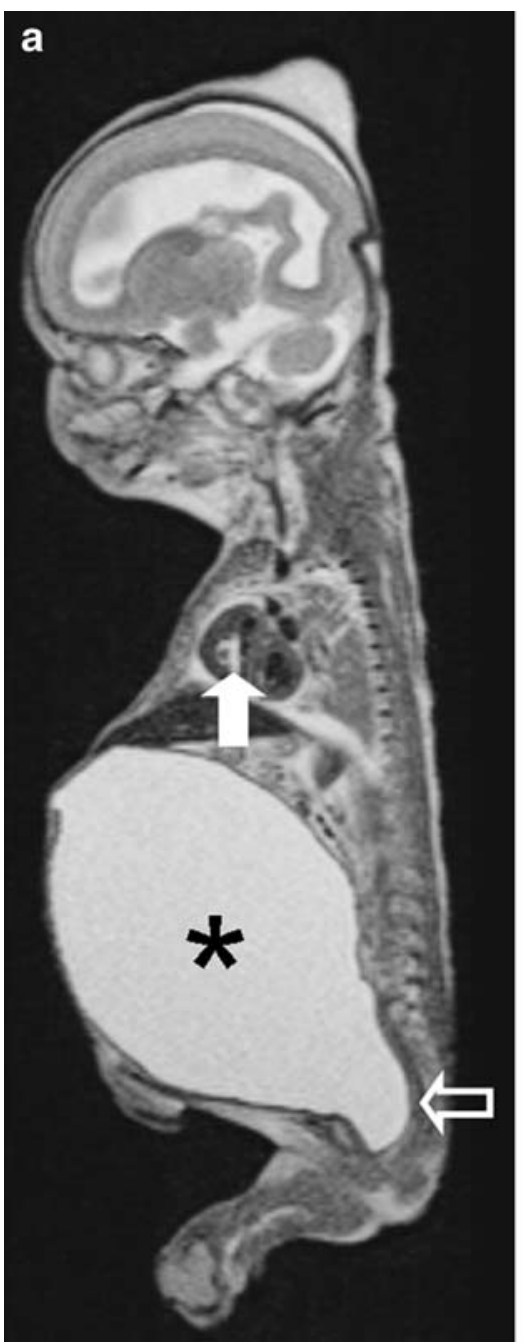

Fig. 9 A neonate aborted at 20 weeks gestational age. a Antenatal US showed a massively dilated bladder and bilateral hydronephrosis. Sagittal T2-W MRI shows a distended bladder (asterisk) and a dilated posterior urethra (open arrow), consistent with posterior urethral valves (slice thickness: $1 \mathrm{~mm}$, TR: 1500, TE: 161, FA: $150^{\circ}$ ). Note the

clinical information is not always available (e.g. in the case of the body of an unknown child).

One of the aims of postmortem forensic radiology is to detect the presence of foreign bodies, such as fragments of glass and bullets, and to describe their position and, if applicable, the trajectory they followed. Radiology will also be able to detect more subtle cases of pneumothorax or pneuperitoneum, for example, which can be missed in a conventional autopsy (Fig. 10). Furthermore, if radiology is used to describe the corpse prior to autopsy it makes revision possible even after the corpse has been buried. In legal proceedings this is an important advantage of postmortem radiology over the conventional autopsy.

There are numerous forensic institutes in the world that have a radiological facility or are contemplating buying a $\mathrm{CT}$ and/or MR scanner $[49,50]$. In Europe one of the most

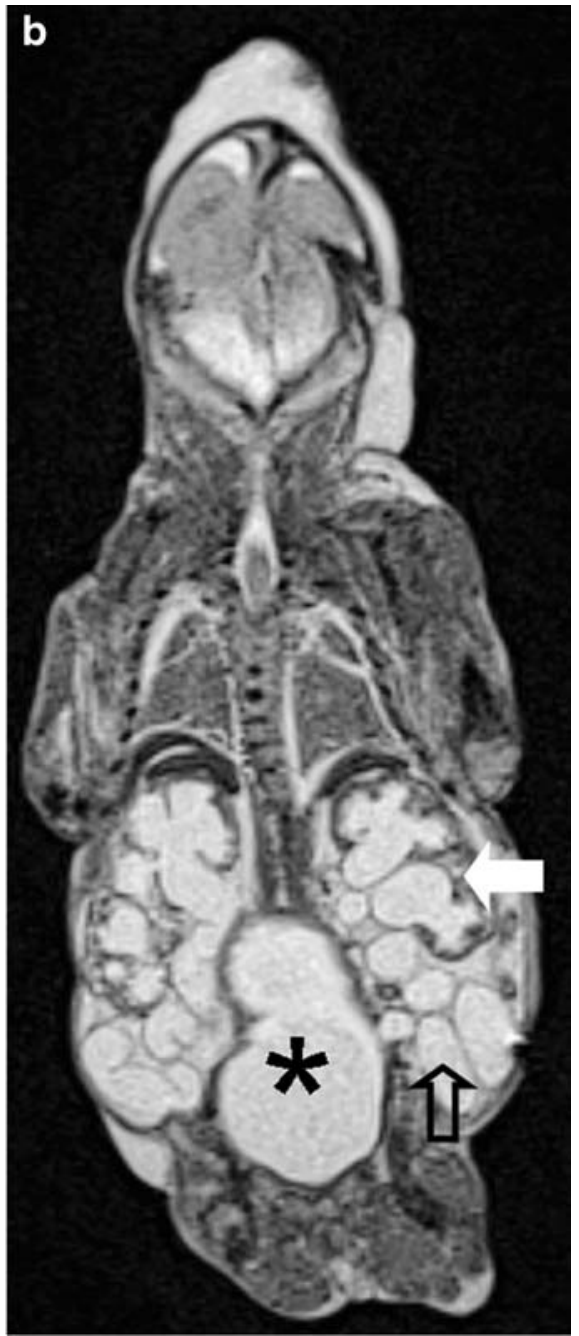

fluid-fluid level in the heart (arrow) as a result of clotting blood. b Coronal T2-W MRI shows a distended bladder (asterisk) and dilated tortuous ureters (open arrow). There is a substantial bilateral pyelocaliceal dilatation (arrow). There is a relative hypoplasia of the lungs as a result of the oligohydramnion

well known is the Forensic Institute and the Centre for Forensic Imaging at the University of Bern, Switzerland [39]. The Virtopsy ${ }^{\circledR}$ project began at the institute, which is one of the leaders in scientific research and development in forensic radiology [51].

It is important to remember that in the end the aim of all forensic necroscopic examinations is to determine the cause (e.g. hypoxia) and manner (e.g. strangulation) of death in order to decide whether a crime has been committed (Fig. 11).

\section{Historical paediatric specimens}

Although slightly outside the scope of postmortem paediatric radiology, the use of radiological imaging of historical 
Fig. 10 A 10-year-old boy who died in the hospital after a fall. a Postmortem CT shows a small pneumothorax, which was not found at autopsy. There is diffuse airway consolidation in keeping with postmortem pulmonary oedema. b Surfaceshaded rendering of the thorax shows an incorrectly positioned left subclavian line with the tip of the line in the jugular vein (arrow). The line was cut and the distal end (arrowhead) was buried subcutaneously
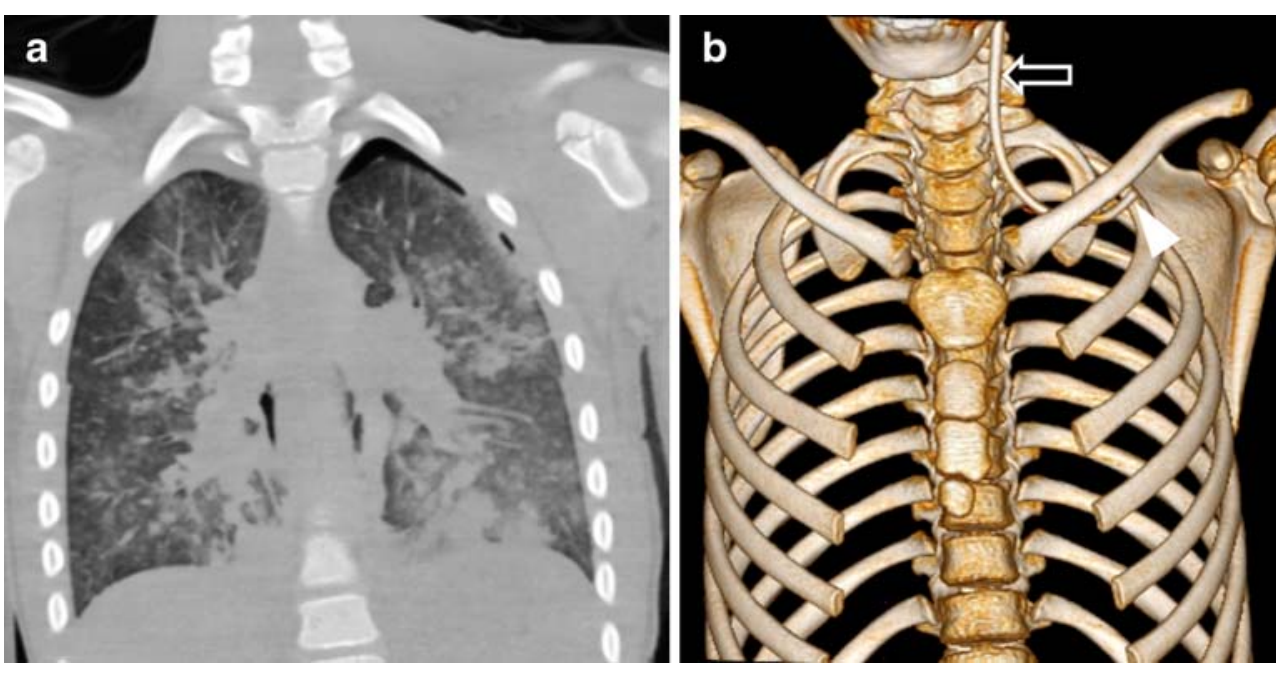

paediatric specimens is worth mentioning. There are many collections of human historical specimens that have been gathered over the centuries by both historians and clinicians. Some of these cases depict diseases and/or disorders that are now very rare and therefore of interest. Radiology can be an excellent tool used to investigate these delicate specimens without destroying them. In this article the use of radiology in this interesting scientific field is demonstrated with two paediatric cases.

The first case is a specimen owned by the Academic Medical Centre Amsterdam, which is home to one of the largest teratological collections in Europe (the Vrolik Museum). This collection of more than 2,000 specimens was founded by Gerardus Vrolik (1775-1859) and his son Willem Vrolik (1801-1863). It shows various aspects of human and animal anatomy, embryology, pathology, and congenital anomalies [52]. One of the specimens in this collection is a cephalothoracopagus, estimated to be 100 150 years old (Fig. 12). Conjoined twins are classified according to the site of union by using the suffix pagus (fixed), and therefore a cephalothoracopagus is joined by the head, thorax and (part of the) abdomen.

The second case is from the National Museum of Antiquities in Leiden, the Netherlands. This particular mummy is of a boy, estimated to be 9.5-14.5 years of age, and has been dated to the 3rd century A.D. (Fig. 13) [53]. The mummy is one of eight (located in several museums worldwide), forming a homogeneous group based on similarities of both the exterior as well as the embalming techniques [53].
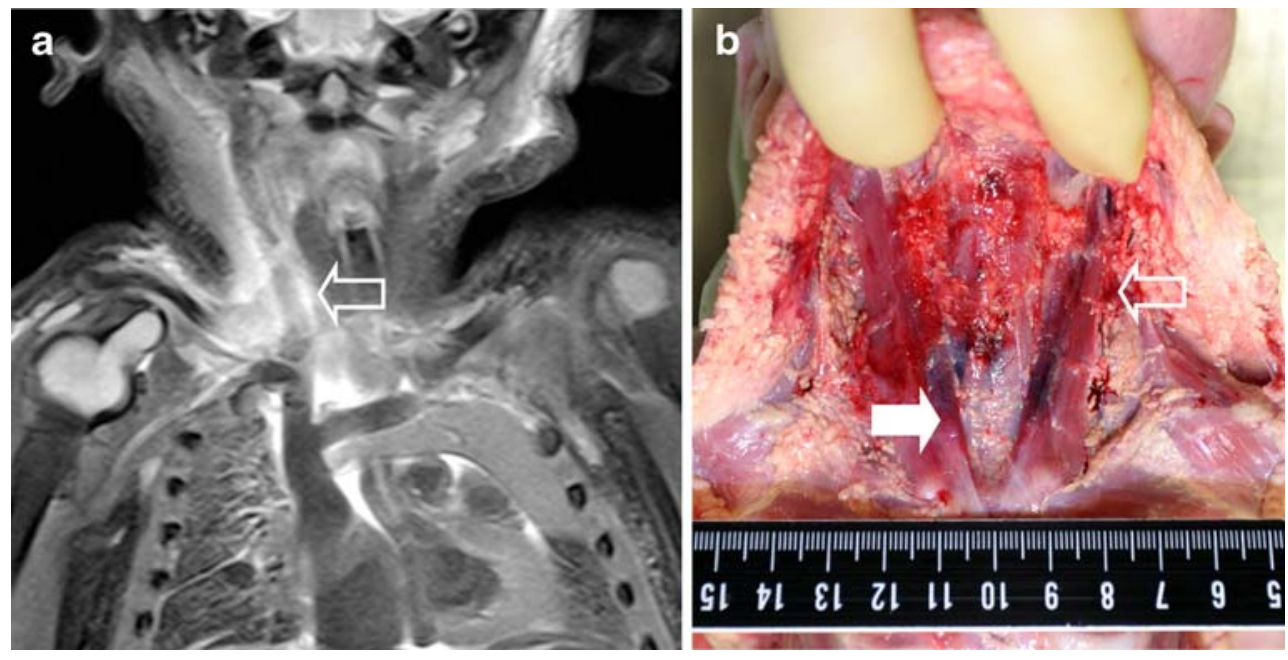

Fig. 11 A neonate of unknown gestational age found in a garbage bin. a Coronal T2-W image shows oedema around the right jugular vein (arrow) (slice thickness: $4 \mathrm{~mm}$, TR: 5970, TE: 84, FA: $150^{\circ}$ ) (Reprinted with permission from Bilo RA, Robben SG, van Rijn RR [2009] Differentiating accidental trauma from child abuse. In:
Forensic aspects of pediatric fractures. Springer-Verlag, in press). b Autopsy shows a bilateral haematoma (open arrow) around the jugular vein, the carotid artery and the sternocleidoid muscles (arrow). This finding is fitting with strangulation 

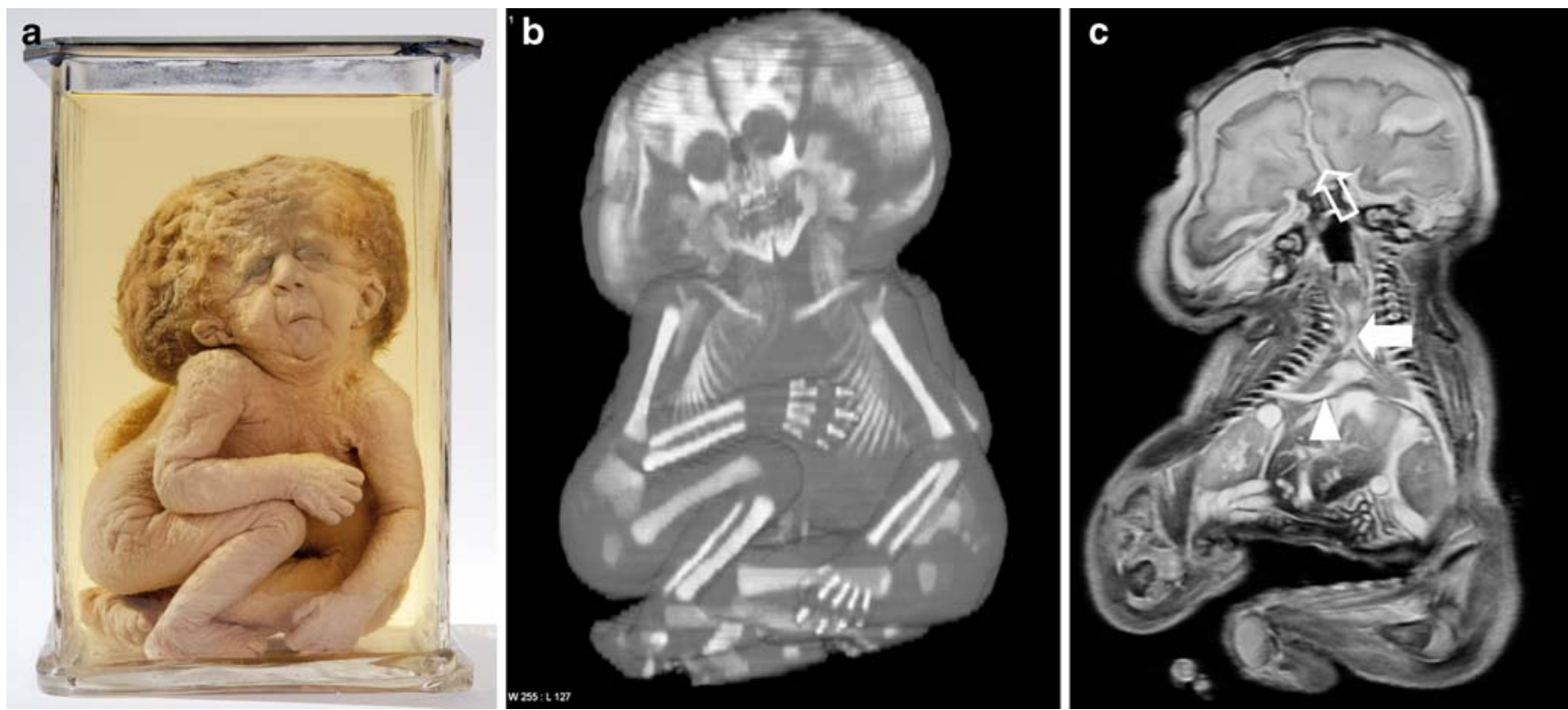

Fig. 12 Historical paediatric specimen. a Image of cephalothoracopagus from the Vrolik Museum. Specimen is estimated to be 100 150 years old. b Surface-shaded rendering shows a conjoined skull and chest; the spine, pelvis and extremities are separate. c Coronal T2-W MRI shows individual development of the brain with a clear separation between the right and left side of the craniothoracophagus

\section{Conclusion}

In this review paper we have presented the current state of postmortem imaging in children. This exciting new field in paediatric radiology opens new areas in which (open arrow) (slice Thickness: $3 \mathrm{~mm}$, TR: 2500, TE: 68, FA: $90^{\circ}$ ). The trachea is fused (arrow) and a single diaphragm is present (arrowhead). There is a compound liver (neoaxial orientation), which on further imaging shows two separate gallbladders. Normal renal development is present

close collaboration between radiologists and pathologists is essential. It is important that paediatric radiologists become involved in this field as pathologists are not trained in reading radiographs and may therefore miss essential clues [54].
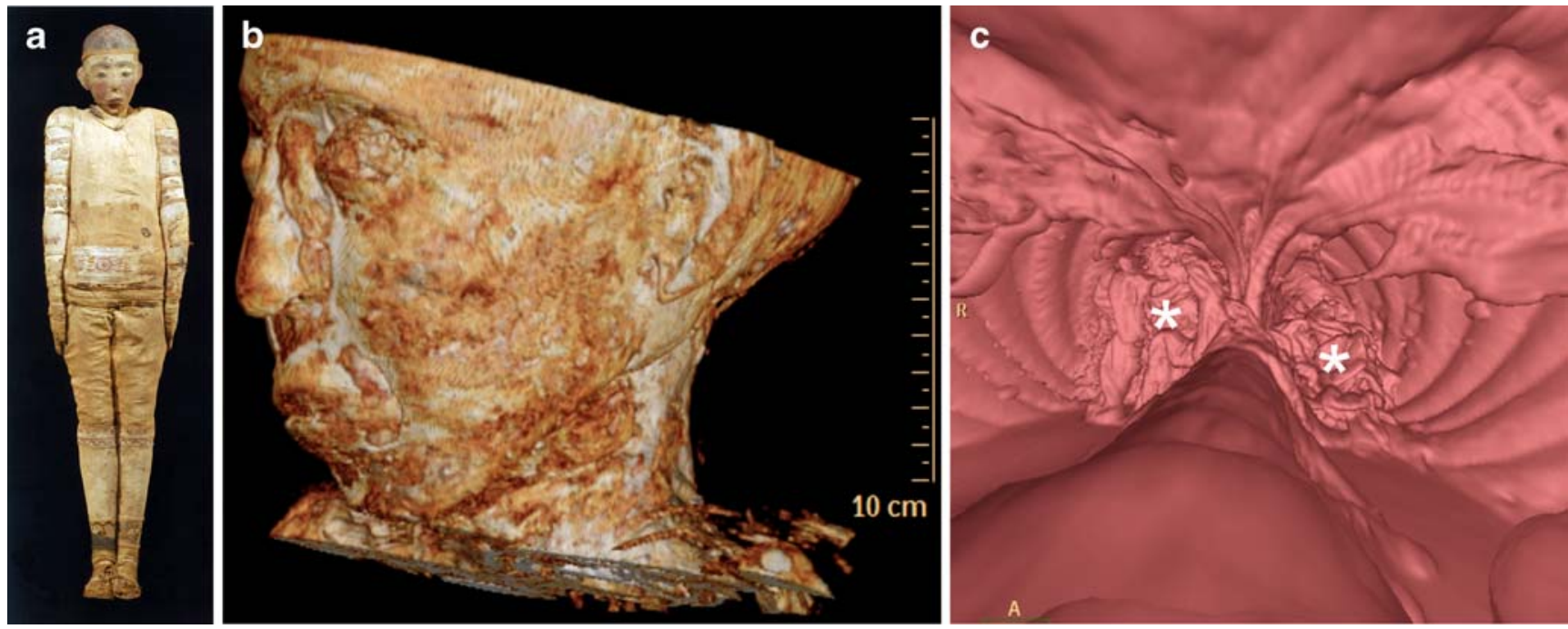

Fig. 13 Second historical specimen. a Mummy of a boy, estimated age 9.5-14.5 years, dated to the 3rd century A.D. b Shaded-surface rendering of the face shows facial features. The nose is slightly depressed, likely as a result of mummification. The ear is relatively large and stands off the skull. c Virtual endoscopy of the abdominal cavity shows absence of both abdominal and thoracic organs. The thorax is partially filled with gauzes (asterisk) (courtesy of the National Museum of Antiquities, Leiden, the Netherlands, reprinted with permission from Raven MJ, Taconis WK (eds) [2005] Egyptian mummies: radiological atlas of the collections in the national museum of antiquities in Leiden. Brepols, Turnhout, Belgium, pp 191-195) 
To date, nearly all radiological studies have looked at either general findings in a relatively small population or specific pathology in larger samples, and in almost all studies the setup was descriptive. The lack of substantially large studies with statistical power and the wide variety of study designs makes it difficult to perform a meta-analysis of the published data. This is clearly illustrated by a recent systematic review by Scholing et al. [55] of the role of postmortem CT in trauma victims. They included 15 studies with a moderate sample size of 13 patients and a range of agreement between postmortem CT and autopsy of $46-100 \%$.

Sebire [56] adds that besides the lack of large-scale studies comparing imaging-based versus autopsy-based diagnoses, data on the accuracy of postmortem-obtained histopathological material has not been published. The combination of postmortem imaging with needle-core biopsy is something that deserves further attention, as most information at a conventional autopsy comes from microscopic histopathological examination. These publications in relation to the diminishing number of autopsies underscore the need for multi-institutional prospective studies in order to assess the full potential of this technique.

It is difficult to predict the future, but it seems certain that radiological techniques will play an important role in the future of both clinical and forensic pathology. For paediatric radiologists involved in this field, it completes the circle of life, making it one of the few medical specialties that cares for patients from the cradle to the grave. Perhaps in the future a new subspecialty of forensic radiology will emerge [57].

Open Access This article is distributed under the terms of the Creative Commons Attribution Noncommercial License which permits any noncommercial use, distribution, and reproduction in any medium, provided the original author(s) and source are credited.

\section{References}

1. Shojania KG, Burton EC, McDonald KM et al (2002) The autopsy as an outcome and performance measure. Evid Rep Technol Assess (Summ) 58:1-5

2. Shojania KG, Burton EC (2008) The vanishing nonforensic autopsy. N Engl J Med 358:873-875

3. Burton JL, Underwood J (2007) Clinical, educational, and epidemiological value of autopsy. Lancet 369:1471-1480

4. Maniscalco WM, Clarke TA (1982) Factors influencing neonatal autopsy rate. Am J Dis Child 136:781-784

5. Newton D, Coffin CM, Clark EB et al (2004) How the pediatric autopsy yields valuable information in a vertically integrated health care system. Arch Pathol Lab Med 128:1239-1246

6. Brodlie M, Laing IA, Keeling JW et al (2002) Ten years of neonatal autopsies in tertiary referral centre: retrospective study. BMJ 324:761-763

7. Snowdon C, Elbourne DR, Garcia J (2004) Perinatal pathology in the context of a clinical trial: attitudes of neonatologists and pathologists. Arch Dis Child Fetal Neonatal Ed 89:F204-F207
8. Sinard JH (2001) Factors affecting autopsy rates, autopsy request rates, and autopsy findings at a large academic medical center. Exp Mol Pathol 70:333-343

9. Hinchliffe SA, Godfrey HW, Hind CR (1994) Attitudes of junior medical staff to requesting permission for autopsy. Postgrad Med J 70:292-294

10. McPhee SJ (1996) Maximizing the benefits of autopsy for clinicians and families. What needs to be done. Arch Pathol Lab Med 120:743-748

11. Dorff EN (2005) End-of-life: Jewish perspectives. Lancet 366:862-865

12. Geller SA (1984) Religious attitudes and the autopsy. Arch Pathol Lab Med 108:494-496

13. Griffiths PD, Paley MN, Whitby EH (2005) Post-mortem MRI as an adjunct to fetal or neonatal autopsy. Lancet 365:1271-1273

14. Burton JL, Underwood JC (2003) Necropsy practice after the "organ retention scandal": requests, performance, and tissue retention. J Clin Pathol 56:537-541

15. Burton JL, Wells M (2001) The Alder Hey affair: implications for pathology practice. J Clin Pathol 54:820-823

16. Khong TY, Tanner AR (2006) Foetal and neonatal autopsy rates and use of tissue for research: the influence of 'organ retention' controversy and new consent process. J Paediatr Child Health 42:366-369

17. Khong TY, Arbuckle SM (2002) Perinatal pathology in Australia after Alder Hey. J Paediatr Child Health 38:409-411

18. McHaffie HE, Fowlie PW, Hume R et al (2001) Consent to autopsy for neonates. Arch Dis Child Fetal Neonatal Ed 85:F4F7

19. Roulson J, Benbow EW, Hasleton PS (2005) Discrepancies between clinical and autopsy diagnosis and the value of post mortem histology; a meta-analysis and review. Histopathology 47:551-559

20. Shojania KG, Burton EC, McDonald KM et al (2003) Changes in rates of autopsy-detected diagnostic errors over time: a systematic review. JAMA 289:2849-2856

21. Pastores SM, Dulu A, Voigt L et al (2007) Premortem clinical diagnoses and postmortem autopsy findings: discrepancies in critically ill cancer patients. Crit Care 11:R48

22. Gordijn SJ, Erwich JJ, Khong TY (2002) Value of the perinatal autopsy: critique. Pediatr Dev Pathol 5:480-488

23. Kumar P, Taxy J, Angst DB et al (1998) Autopsies in children: are they still useful? Arch Pediatr Adolesc Med 152:558-563

24. Wright C, Lee RE (2004) Investigating perinatal death: a review of the options when autopsy consent is refused. Arch Dis Child Fetal Neonatal Ed 89:F285-F288

25. Cohen MC, Paley MN, Griffiths PD et al (2008) Less invasive autopsy: benefits and limitations of the use of magnetic resonance imaging in the perinatal postmortem. Pediatr Dev Pathol 11:1-9

26. Whitby EH, Paley MN, Cohen M et al (2005) Postmortem MR imaging of the fetus: an adjunct or a replacement for conventional autopsy? Semin Fetal Neonatal Med 10:475-483

27. American College of Radiology (ACR) (2006) ACR practice guideline for skeletal surveys in children. Available via http:// www.acr.org/SecondaryMainMenuCategories/quality_safety/ guidelines/pediatric/skeletal_surveys.aspx. Accessed 13 Nov 2009

28. The Royal College of Radiologists and the Royal College of Paediatrics and Child Health (2008) Standards for radiological investigations of suspected non-accidental injury. Available via http://www.rcr.ac.uk/docs/radiology/pdf/RCPCH_RCR_final.pdf. Accessed 13 Nov 2009

29. Offiah A, van Rijn RR, Perez-Rossello JM et al (2009) Skeletal imaging of child abuse (non-accidental injury). Pediatr Radiol 9:461-470

30. Grabherr S, Dirnhofer R (2009) Postmortem angiography. In: Thali MJ, Dirnhofer R, Vock P (eds) The Virtopsy approach: 3D 
optical and radiological scanning and reconstruction in forensic medicine. CRC, Boca Raton, pp 443-450

31. Stoeter P, Voigt K (1976) Radiological examination of embryonal and fetal vessels. Technique and method of prenatal, postmortem angiography in different stages of gestation. Rofo 124: $558-564$

32. Farina J, Millana C, Fdez-Acenero MJ et al (2002) Ultrasonographic autopsy (echopsy): a new autopsy technique. Virchows Arch 440:635-639

33. Uchigasaki S, Oesterhelweg L, Gehl A et al (2004) Application of compact ultrasound imaging device to postmortem diagnosis. Forensic Sci Int 140:33-41

34. Ross S, Spendlove D, Bolliger S et al (2008) Postmortem wholebody CT angiography: evaluation of two contrast media solutions. AJR 190:1380-1389

35. Kleinman PK, Marks SC Jr, Nimkin K et al (1996) Rib fractures in 31 abused infants: postmortem radiologic-histopathologic study. Radiology 200:807-810

36. OE OE, Espeland A, Maartmann-Moe H et al (2003) Diagnostic value of radiography in cases of perinatal death: a population based study. Arch Dis Child Fetal Neonatal Ed 88:F521-F524

37. Seppanen U (1985) The value of perinatal post-mortem radiography. Experience of 514 cases. Ann Clin Res 17(Suppl 44):1-59

38. Foote GA, Wilson AJ, Stewart JH (1978) Perinatal post-mortem radiography-experience with 2500 cases. Br J Radiol 51:351-356

39. Bolliger SA, Thali MJ, Ross S et al (2008) Virtual autopsy using imaging: bridging radiologic and forensic sciences. A review of the Virtopsy and similar projects. Eur Radiol 18:273-282

40. Huisman TA (2004) Magnetic resonance imaging: an alternative to autopsy in neonatal death? Semin Neonatol 9:347-353

41. Woodward PJ, Sohaey R, Harris DP et al (1997) Postmortem fetal MR imaging: comparison with findings at autopsy. AJR 168:41-46

42. Griffiths PD, Variend D, Evans M et al (2003) Postmortem MR imaging of the fetal and stillborn central nervous system. AJNR $24: 22-27$

43. Breeze AC, Cross JJ, Hackett GA et al (2006) Use of a confidence scale in reporting postmortem fetal magnetic resonance imaging. Ultrasound Obstet Gynecol 28:918-924

44. Alderliesten ME, Peringa J, van der Hulst VP et al (2003) Perinatal mortality: clinical value of postmortem magnetic resonance imaging compared with autopsy in routine obstetric practice. BJOG 110:378-382
45. Thayyil S, Cleary JO, Sebire NJ et al (2009) Post-mortem examination of human fetuses: a comparison of whole-body high-field MRI at 9.4 $\mathrm{T}$ with conventional MRI and invasive autopsy. Lancet 374:467-475

46. Weustink AC, Hunink MG, van Dijke CF et al (2009) Minimally invasive autopsy: an alternative to conventional autopsy? Radiology 250:897-904

47. Yen K, Lovblad KO, Scheurer E et al (2007) Post-mortem forensic neuroimaging: correlation of MSCT and MRI findings with autopsy results. Forensic Sci Int 173:21-35

48. Cox J, Kirkpatrick RC (1896) The new photography with report of a case in which a bullet was photographed in the leg. Montreal Med J 24:661

49. Rutty GN, Morgan B, O'Donnell C et al (2008) Forensic institutes across the world place CT or MRI scanners or both into their mortuaries. J Trauma 65:493-494

50. Thomsen AH, Jurik AG, Uhrenholt L et al (2009) An alternative approach to computerized tomography (CT) in forensic pathology. Forensic Sci Int 183:87-90

51. Thali MJ, Braun M, Buck U et al (2005) VIRTOPSY-scientific documentation, reconstruction and animation in forensic: individual and real 3D data based geo-metric approach including optical body/object surface and radiological CT/MRI scanning. J Forensic Sci 50:428-442

52. Baljet B, Oostra RJ (1998) Historical aspects of the study of malformations in The Netherlands. Am J Med Genet 77:91-99

53. Raven MJ, Taconis WK (2005) Egyptian mummies: radiological atlas of the collections in the national museum of antiquities in Leiden. Brepols, Belgium, pp 191-195

54. Kremer C, Racette S, Marton D et al (2008) Radiographs interpretation by forensic pathologists: a word of warning. Am J Forensic Med Pathol 29:295-296

55. Scholing M, Saltzherr TP, Fung Kon Jin PH et al (2009) The value of postmortem computed tomography as an alternative for autopsy in trauma victims: a systematic review. Eur Radiol 10:2333-2341

56. Sebire NJ (2006) Towards the minimally invasive autopsy? Ultrasound Obstet Gynecol 28:865-867

57. O'Donnell C, Woodford N (2008) Post-mortem radiology-a new sub-speciality? Clin Radiol 63:1189-1194

58. Broumandi DD, Hayward UM, Benzian JM et al (2004) Best cases from the AFIP: hemimegalencephaly. Radiographics 24:843-848 